\title{
La résistance au paludisme. Interaction de facteurs multiples
}

Si la notion d'une action à la fois sélective et protectrice exercée par le paludisme sur les hémoglobinopathies repose sur un ensemble incontestable de données épidémiologiques, le mécanisme en cause est encore très mal connu malgré le grand nombre d'études focalisées sur le sujet. Un travail récent, impliquant plusieurs équipes, mais coordonné par celle de John Clegg (Oxford, GB) apporte à cette compréhension une contribution importante [1]. La même équipe, étudiant l'épidémiologie du paludisme à Plasmodium falciparum en Mélanésie, avait pu établir une corrélation très étroite, dans laquelle interviennent latitude et altitude, entre endémie palustre et $\alpha$-thalassémie, rendant plus que vraisemblable l'hypothèse d'une sélection par le parasite [2]. Le présent travail, appuyé sur des données de biologie moléculaire, a été mené dans une région du Vanuatu où la fréquence génique de l' $\alpha$-thalassémie délétionnelle est 0,26 , et le paludisme hyperendémique, dû tant au $P$. falciparum, qu'au $P$. vivax. Chez plus de 900 enfants de moins de 10 ans, le génotype a été déterminé et la fréquence d'épisodes cliniques fébriles de paludisme relevée. A leur surprise, les auteurs ont constaté une fréquence nettement accrue $(\mathrm{p}<0,005)$ chez les enfants homozygotes pour la délétion $(\alpha-/ \alpha-)$. Cette prédisposition n'était observée qu'avant 5 ans, elle existait vis-à-vis des deux parasites et s'accompagnait d'une splénomégalie supérieure à celle qu'induit l' $\alpha$-thalassémie seule. Chez les hétérozygotes $(\alpha \alpha / \alpha-)$ la fréquence des épisodes cliniques n'était pas augmentée, mais seule l'était celle d'une splénomégalie. La question se pose donc de $m / s n^{\circ} 1$, vol. 13 , janvier 97 réconcilier cette incidence accrue d'un paludisme à traduction clinique avec la preuve d'une protection contre les formes graves qu'ont apportée les données épidémiologiques. Les auteurs donnent à cette contradiction apparente une base immunologique. Comme dans tous les syndromes thalassémiques, il y a au cours des $\alpha$-thalassémies un cèrtain degré d'érythropoïèse inefficace, et donc une augmentation des cellules jeunes dans la circulation qui, métaboliquement plus actives, sont préférentiellement envahies par le $P$. falciparum; quant au $P$. vivax, il n'envahit que les réticulocytes. L'observation, par ailleurs, que la présentation de l'antigène parasitaire au système immunitaire, à la surface des érythrocytes, est double dans le globule rouge $\alpha$-thalassémique infesté de ce qu'elle est dans la cellule normale n'est pas sans intérêt de ce point de vue [3]. Il est loisible de penser que, pendant la petite enfance, l'enfant étant protégé par l'immunité maternelle et par un taux élevé d'hémoglobine fœtale, des infections répétées provoquent une immunité, qui protégera ultérieurement contre les formes graves. L'hypothèse d'une immunité croisée par le $P$. vivax est évoquée, liée à une infestation de l'enfant $\alpha$ thalassémique par ce parasite plus précoce dans l'existence, et apportant une protection limitée mais réelle contre les formes graves ultérieures dues à $P$. falciparum [4]. Cependant, cette hypothèse semble en contradiction avec le fait que le $P$. vivax, qui a eu des usages thérapeutiques, ne s'est pas toujours avéré protecteur contre le P. falciparum.

Les phénomènes de résistance au paludisme, et la sélection qui en est la conséquence, sont d'importance majeure. L'équipe d'Oxford avait montré le rôle de certains antigènes HLA $\left(\mathrm{m} / \mathrm{s} n^{\circ} 9\right.$, vol 7, p. 981) et ultérieurement identifié à la phase hépatique du parasite un nonapeptide reconnu spécifiquement par les lymphocytes $T$ cytotoxiques spécifiques de HLA-B53 et pouvant servir de base à une vaccination [5]. Le rôle régulateur de cytokines a aussi pu être montré: une mutation du promoteur du TNF- $\alpha$ est lié en Gambie à une susceptibilité au paludisme cérébral [6]. Par conséquent, les mécanismes de la sensibilité ou de la résistance au paludisme sont, à l'évidence, plurigéniques, nécessitant des recherches pluridisciplinaires et complexes, amplement justifiées par la gravité de cette maladie et les perspectives attendues des progrès accomplis.

D.L.

1. Williams TN, Maitland K, Bennett S, Ganczakowski M, Peto TEA, et al. High incidence of malaria in $\alpha$-thalassemic children. Nature 1996; 383: $522-5$.

2. Flint J, Hill AV, Bowden DK, Oppenheimer SJ, Sill PR, et al. High frequencies of alpha-thalassaemia are the result of natural selection by malaria. Nature 1986; 321 : 744-50.

3. Luzzi GA, Merry AH, Newbold CI, Marsh K, Pasvol G, Weatherall DJ, et al. Surface antigen expression on Plasmodium falciparum-infected erythrocytes is modified in alpha- and beta-thalassemia. J Exp Med 1991 ; 173 : 785-91.

4. Miller LH. Protective selective pressure. Nature $1996 ; 383: 480-1$.

5. Hill AVS, Elvin J, Willis AC, Aidoo M, Allsopp $\mathrm{CE}$, et al. Molecular analysis of the association of HLA-B53 and resistance to severe malaria. Nature 1992 ; 360 : 434-9.

6. McGuire W, Hill AVS, Allsopp CE, Greenwood $\mathrm{BM}$, Kwiatkowski D. Variation in the TNF- $\alpha$ promoter region associated with susceptibility to cerebral malaria. Nature 1994 ; 371 : 508-11. 